
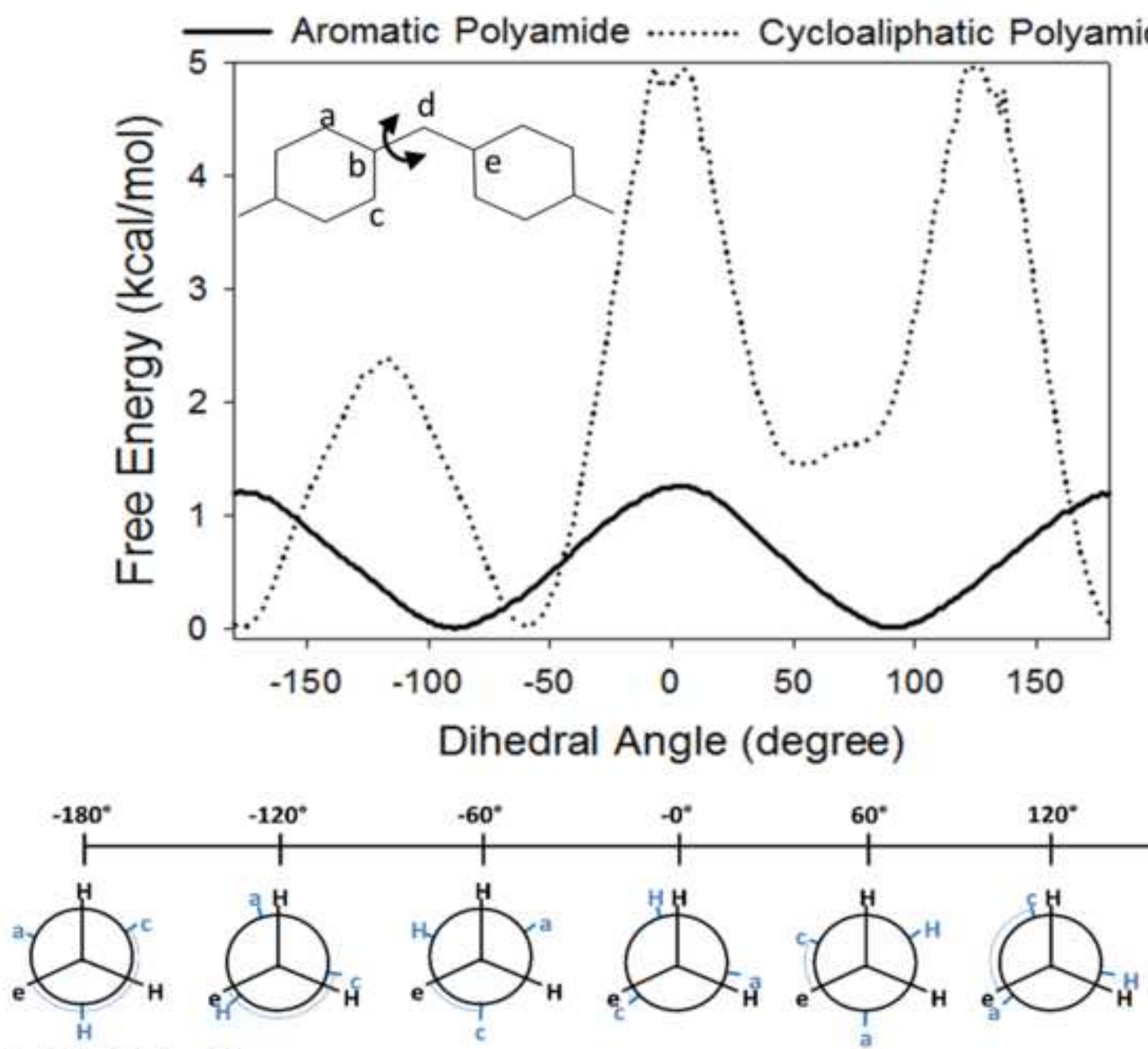

Cycloaliphatic Polyamide 


\title{
Investigating the glass transition temperature at the atom-level in select model polyamides:
}

\author{
A molecular dynamics study \\ Tanya L Chantawansri, In-Chul Yeh, and Alex J Hsieh \\ US Army Research Laboratory, Aberdeen Proving Ground, MD 21005
}

\begin{abstract}
The molecular influence on glass transition temperature $\left(T_{g}\right)$ of select model polyamides was investigated by atomistic modeling. These include nylon 6,6, nylon 6,12, a cycloaliphatic polyamide consisting of 4,4'-methylene-bis(cyclohexylamine) and dodecanedioic acid, as well as a corresponding aromatic counterpart consisting of 4,4'-methylenedianiline and dodecanedioic acid. For each model system, all-atom atomistic molecular dynamics simulations were used to discern and differentiate parameters such as free volume, hydrogen bonding, and chain rigidity. Simulations were able to predict the correct trend in $T_{g}$, where the model cycloaliphatic polyamide was shown to have the highest $T_{g}$ followed by the aromatic polyamide. This is presumably due to the greater chain rigidity exhibited by the cycloaliphatic polyamide around the cyclohexyl ring, which was revealed through a calculation of the dihedral rotation free energy barrier associated with the methylene moiety between either the bis-cyclohexyl or bisphenyl rings, despite the former having a higher extent of free volume.
\end{abstract}

\section{Introduction}

An important concept for polymers is the glass transition temperature, $T_{g}$, which plays a crucial role in both the physical and mechanical properties including strength, toughness, creep, and stiffness[1]. The $T_{g}$ is typically observed in amorphous materials, or in the vicinity of amorphous regions in semi-crystalline polymers. Even so, the presence of crystalline phases will affect the orientation and segmental mobility of surrounding amorphous regions thus indirectly affecting the $T_{g[2]}$. The $T_{g}$ involves a shift in the temperature derivatives, heat capacity, and coefficients of expansion, which are all dependent on the molecular motion of the polymer[3].

One factor that can affect the segmental motion of polymers is the amount of free volume or empty space available to accommodate the relaxation of polymer chains. On a molecular level, the concept of free volume was derived to interpret molecular motions in the bulk state, where the presence of holes or microvoids would be required for local motion such as rotation, 
or cooperative motion including reputation[4]. From the kinetics perspective, vitrification upon supercooling of polymers to the glassy state, where the mismatch between the polymer chain relaxation and supercooling rates would lead to the entrapment of unoccupied volume and the formation of free volume in polymers. The extent of free volume depends on a variety of factors such as chain flexibility (including stiffness and to a lesser extent the size of side chains), geometric factors (such as symmetry of backbone and presence of double bond on the main chains), and the number of chain ends.

Typically, polymers with high chain rigidity and less free volume have higher $T_{g}$, but these two characteristics do not always occur concurrently. Li et al.[5] observed that isomeric polyimides (PI) had a higher $T_{g}$ than its corresponding symmetrical analogue. Isomeric PI exhibits a higher free volume fraction, but the torsion angle of the bi-phenyl group was constrained thus leading to an increase in the rigidity of polymer chains. The extent of hydrogen bonding is also another factor that can also indirectly affect both the free volume and chain rigidity/mobility. Hydrogen bonding restricts molecular motion through the formation of temporary "cross-links", which can result in an increase in the $T_{g}[6,7]$.

These factors all affect the $T_{g}$ of synthetic polyamides, which are a class of high performance engineering materials that exhibit good mechanical properties. Aliphatic polyamides like nylons have a broad range of applications among which nylon 6 and nylon 6,6 are of great commercial importance in fibers and engineering plastics[8-10]. On the other hand, aromatic Kevlar materials provide outstanding tensile strength-to-weight ratio properties, about five times stronger than steel, and have various applications in personnel protection such as combat helmets, ballistic vests, and spall liners for armored vehicles[11, 12]. Although their use is widespread, challenges remain for synthetic polyamides, where despite their versatile 
chemistries, a better understanding of how the material behaves at the molecular level is critical to the design of materials that exhibit an optimal microstructure that corresponds to desirable physical and mechanical properties.

In this work, atomistic all-atom simulations were performed to calculate the $T_{g}$ of model polyamide systems. In atomistic modeling, chemical details of the different chemical species in the polymer are captured through atomistic force fields, which are crucial for an accurate description of structural and dynamical properties of polyamides containing polar amide groups. Four different systems were considered: nylon 6,6, nylon 6,12, a model cycloaliphatic polyamide and the corresponding aromatic analog. In this work, our focus is to discern and reveal the role of the cycloaliphatic conformation vs. the corresponding aromatic counterpart on intermolecular hydrogen bonding, free volume and $T_{g}$ in select model polyamides. Results will be compared to the more traditional aliphatic polyamides such as nylon 6,6 and 6,12 .

\section{Model Systems}

Four different polyamide systems were considered: nylon 6,6, nylon 6,12, a select model cycloaliphatic polyamide consisting of 4,4'-methylene-bis(cyclohexylamine) and dodecanedioic acid, and a corresponding aromatic counterpart consisting of 4,4'-methylenedianiline and dodecanedioic acid. For brevity, the two model polyamides are labeled cycloaliphatic polyamide and aromatic polyamide, respectively. Their chemical structures are shown in Figure 1. 


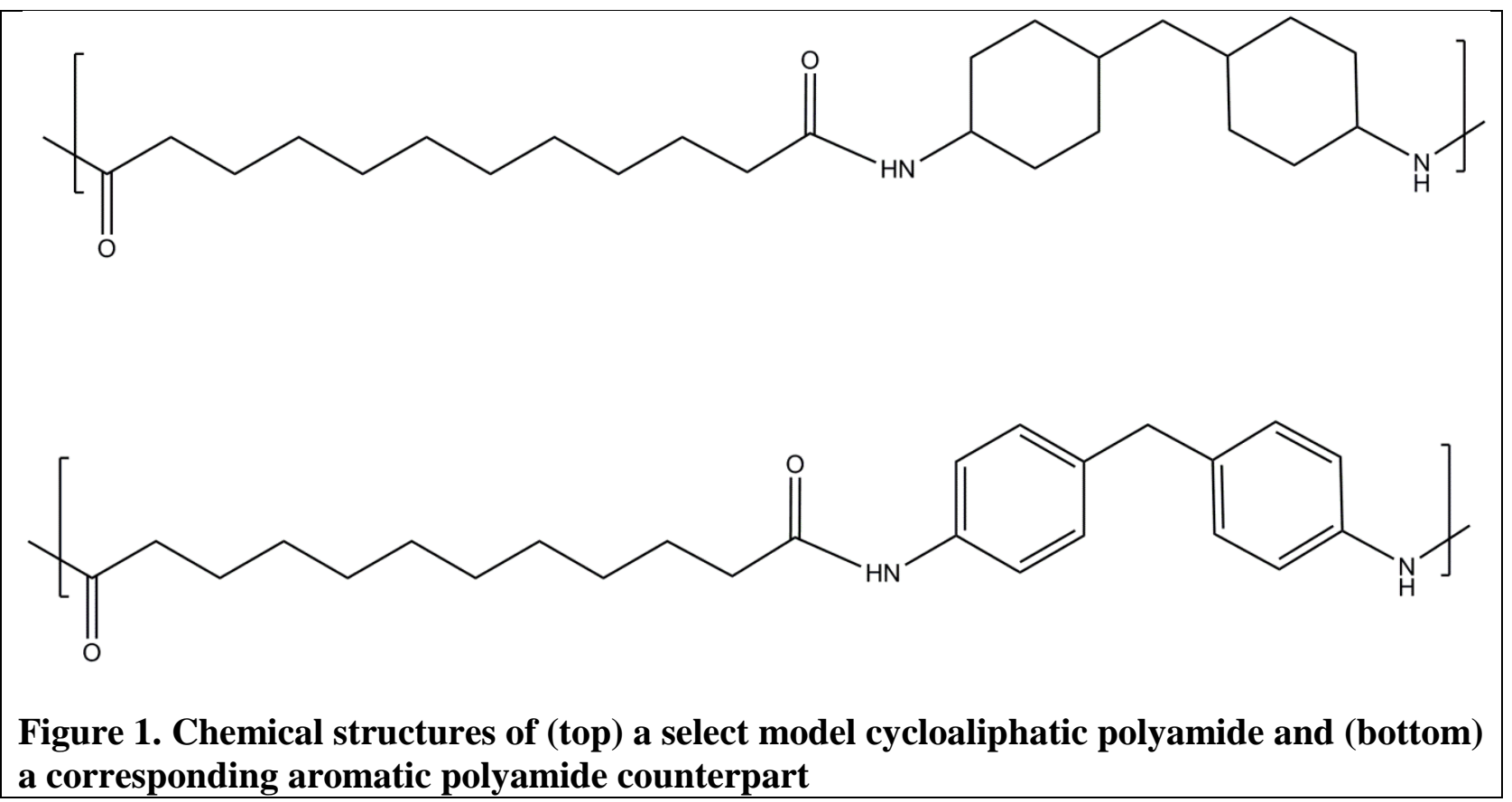

Traditionally, molecules with greater propensity towards (long-range) ordering or an increase in intermolecular association such as intermolecular hydrogen bonding were thought to favor the formation of crystalline microstructure in semicrystalline polymers. Polymers containing aromatic rings, for example, are often regarded as being more rigid and through aromatic stacking interactions between the adjacent chains they are more prone to crystallization. This stacking interaction is observed experimentally via both wide-angle X-ray scattering and differential scanning calorimetry (DSC) measurements in polymers, such as aromatic polyamides like poly(paraphenylene terephthalamide)[13], aromatic polyesters including polybutylene terephthalate[14] and polyethylene terephthalate[15], as well as in 4,4'-methylene diphenyl diisocyanate (MDI) based polyurethanes[16]. It was also noted that the diffraction patterns of the chain structure in a 4,4'-MDI-butanediol based polyurethane resembled the $\alpha$-form crystalline structure of nylon $6,6[17,18]$. As for the select model aliphatic and aromatic polyamides, DSC results revealed the presence of multiple endothermic peaks in the aromatic 
4,4'-methylenedianiline based polyamide, indicative of a stronger tendency for crystallization, in contrast to the corresponding cycloaliphatic polyamide[19].

The time and length scales accessible to all-atom molecular dynamics is on the order of nanoseconds and of angstroms, which is not sufficient to capture phenomenon such as phase separation and the formation of crystalline regions[20]. Even so, this modeling method can be used to study the fully amorphous system, where the effect of chemistry on the $T_{g}$, as well as on the extent of hydrogen bonding, chain rigidity, and free volume, will be discerned. Although the effect of the crystalline region will be ignored which will affect the segmental mobility near the amorphous-crystalline interface[21], information obtained from the purely amorphous region can still help elucidate aspects that affect the overall $T_{g}$ of the material.

\section{Simulation Models and Methods}

Initial cubic simulation cells were constructed using the amorphous cell module of Materials Studio 6.0 (Accelrys Inc.[22]). This method uses a Monte-Carlo technique to build an amorphous structure of the polymer by using rotational isomeric state theory and taking into account nonbonded interactions with the already constructed neighboring chains, including their periodic images[23]. Each simulation cell contained 30 chains, each with 10 repeat units, where the polymer consistent force field (PCFF[24]) was used to describe the interactions between the different atoms. The partial charges were taken from the condensed-phase optimized molecular potentials for atomistic simulation studies (COMPASS[25]) force field. Three replicas were considered for each model polyamide, where systems were built to be amorphous with no prescribed crystallinity.

The nonbonded van der Waals interactions were described by a 9-6 Lennard Jones

potential, with a cutoff of $12 \AA$, while the nonbonded long-range electrostatic interaction was 
captured through the use of the particle-particle, particle-mesh method[26] with a precision of $10^{-6}$. Temperature and pressure were controlled using the Nose-Hoover thermostat with a $100 \mathrm{fs}$ coupling constant and the Nose-Hoover barostat with a 1 ps coupling constant, respectively, using a time step of $1 \mathrm{fs}$. Simulations were performed using the Large-scale Atomic/Molecular Massively Parallel Simulator (LAMMPS)[27, 28].

Systems were initially relaxed with energy minimization. NVE (constant, particle, volume, and energy) simulations with varying time steps and several cycles of annealing were then performed. After annealing the simulation cell was allowed to equilibrate under constant temperature and pressure (NPT) at a temperature of $700 \mathrm{~K}$ and a pressure of $1 \mathrm{~atm}$ for $4 \mathrm{~ns}$. For the NPT simulations, the three diagonal components were coupled together when the pressure was computed, and the dimensions dilated and contracted in concert therefore preserving the initial cubic cell geometry. A temperature scan was then performed where the temperature is decreased from $700 \mathrm{~K}$ to $100 \mathrm{~K}$ at $20 \mathrm{~K}$ intervals a rate of $10 \mathrm{~K} / \mathrm{ns}$ to produce a temperature vs. specific volume plot from which $T_{g}$ was extracted. The simulated cooling rate is orders of magnitude faster than those used experimentally, which will influence the structure and consequently the properties calculated making direct comparison with experimental values difficult. Even so, molecular modeling can be used to provide qualitative understanding, where possible mechanisms that can influence the properties of the material can be explored. Values of the specific volume were obtained from a batch average of the last 1 ns. Trajectories during the temperature scan were also used for calculations of free volume and hydrogen bonding numbers and lifetimes.

Computed trajectories were subsequently analyzed to determine the role of chemistry and temperature on the formation of a hydrogen bond network and the free volume. In our 
calculation, we considered a hydrogen bond to have formed between an amide hydrogen atom and an oxygen atom if the distance between the two atoms was less than $3 \AA$ and also if the angle $\angle \mathrm{N}-\mathrm{H}$... O was larger than $130^{\circ}$. The formation and dissociation of hydrogen bonds as a function of time was tracked to estimate the lifetime of hydrogen bonding, which is defined as the duration of a continuous hydrogen bond. Average hydrogen bonding lifetimes were obtained by averaging lifetimes over all observed hydrogen bonds. The numbers and lifetimes of hydrogen bonds were calculated for our model polyamide systems by considering the last $1 \mathrm{~ns}$ of the $2 \mathrm{~ns}$ simulation at each temperature, where the simulation coordinates were saved every 1 ps.

The fractional free volume was calculated using the energy-based Cavity Energetic Sizing Algorithm (CESA), which captures the free volume at the local level rather than the Bondi method which captures free volume as a global quantity. CESA[29] is an energy-based rather than geometric-based Monte Carlo technique that characterizes free volume in terms of spherical cavities, each of which has a well-defined center and is a local minimum in a repulsive particle energy field. This method calculates the fractional cavity volume (FCV), which is the fraction of the free volume occupied by spherical cavities. In addition, distributions of these cavities can be calculated to determine the size and dissemination of free volume in the polymer. The FCV calculated through CESA typically captures $25-50 \%$ of the Bondi free volume, and has been shown to agree with free volume measurements obtained from experimental position annihilation lifetime spectroscopy (PALS)[30, 31]. Willmore et al.[32] extended the CESA method through the introduction of shape parameters which have been used to characterize the non-spherical aspects of free volume through the definition of clusters, which are made up of overlapping cavities. Each cavity is considered to be part of only one cluster, where each cluster has at least one cavity. These clusters can be characterized through shape parameters such as 
volume, surface area, span (largest distance between two points in a cluster), and radius of gyration, where these quantities can be used to access the connectivity of the free volume. CESA was used to characterize our polyamide systems as a function of temperatures, where analysis was performed on the last 200 ps of each temperature stage, where snapshots were evaluated every 20 ps. Due to the high computational cost of these free volume calculations, only 10 snapshots were analyzed at each temperature. Even so, calculated quantities were consistent to those obtained by analyzing the last $1 \mathrm{~ns}$ of the simulation, when snapshots were taken every 5 ps.

In addition to studying the effect of temperature, we will also take a closer look at system properties at ambient conditions $(300 \mathrm{~K})$. To access local rigidity, we calculate the free energy or potential-of-mean force $[33,34]$ associated with these rotations around a single $\mathrm{C}-\mathrm{C}$ bond in the bis-cyclohexyl ring and bis-phenyl ring moieties present in the cycloaliphatic and aromatic polyamide, respectively. This quantity can be calculated through $E=-R T \log (P(\psi))$, where $R$ is the ideal gas constant, $T$ is the temperature, and $P(\psi)$ is the probability of observing a dihedral angle, $\psi$. The probabilities were extracted from the last $1 \mathrm{~ns}$ of the $N P T$ simulation. More comprehensive analysis of the density, number of hydrogen bonds, and free volume was also performed. For these calculations the $300 \mathrm{~K}$ simulation during the temperature ramp, rather than the separate $4 \mathrm{~ns} N P T$ simulation prior to the temperature ramp was considered for analysis since it went through additional extensive high-temperature annealing which will produce a more equilibrated structure. Even so, calculations performed using both trajectories are qualitatively consistent.

\section{Results and Discussion}


Simulation trajectories were analyzed to calculate the density and the $T_{g}$ for our model polyamide systems. To study the influence of hydrogen bonding and free volume, we calculated the number and lifetime of hydrogen bonds as a function of temperature, in addition to properties associated with the free volume. Additionally, the dihedral angle associated with structural differences between the cycloaliphatic and aromatic polyamide systems are compared to assess local chain rigidity. Results are an average of three replicas unless otherwise stated.

\section{A. Densities}

Table 1 lists density values calculated under ambient conditions (300K) through atomistic modeling. For systems where experimental data are available, the calculated values are consistently lower (3.8-11.5\% lower), where the smaller value of density could be due to the force field that we utilized which has not been optimized for these materials. Even so, the force field has been shown to adequately reproduce the thermal and physical properties of amorphous polyamides[35]. We are also currently considering purely amorphous configurations, while experimental values were obtained for semicrystalline samples that can contain denser crystalline regions. This would be particularly true for nylon 6,6 and 6,12 , which exhibit a larger percentage of crystallinity than our model cycloaliphatic polyamide. This accounts for the larger difference between the experimental and calculated density in nylon 6,12 compared to the cycloaliphatic polyamide. In addition, effects of physical aging not captured through modeling due to restrictions in simulation time could contribute to an insufficient structural relaxation and a decrease in calculated density[1], which could lead to the observed deviation. 


\section{B. Glass Transition Temperature}

Table 1. Density values obtained from atomistic simulations. Corresponding experimental values are provided for comparison.

\begin{tabular}{|c|c|c|}
\hline Polymer & $\begin{array}{l}\text { Density from } \\
\text { simulation }\left(\mathrm{g} / \mathrm{cm}^{3}\right)\end{array}$ & $\begin{array}{l}\text { Density from } \\
\text { experiment }\left(\mathrm{g} / \mathrm{cm}^{3}\right)\end{array}$ \\
\hline Nylon 6,6 & $1.029 \pm 0.002$ & $1.069[36], 1.09[37]$ \\
\hline Nylon 6,12 & $0.951 \pm 0.002$ & $1.06[38]$ \\
\hline Cycloaliphatic polyamide & $0.952 \pm 0.002$ & $1.02[39]$ \\
\hline Aromatic Polyamide & $1.036 \pm 0.001$ & $\mathrm{n} / \mathrm{a}$ \\
\hline
\end{tabular}

The $T_{g}$ for our model polyamide systems were extracted from a plot of the specific

volume versus temperature data obtained through NPT simulations as shown in Figure 2 and 3.

The density-temperature data exhibit a linear relationship above (rubbery region) and below (glassy region) the $T_{g}$, where the intersection of the two linear fits corresponds to $T_{g}$. Extracted values of $T_{g}$ are shown in Table 2 .

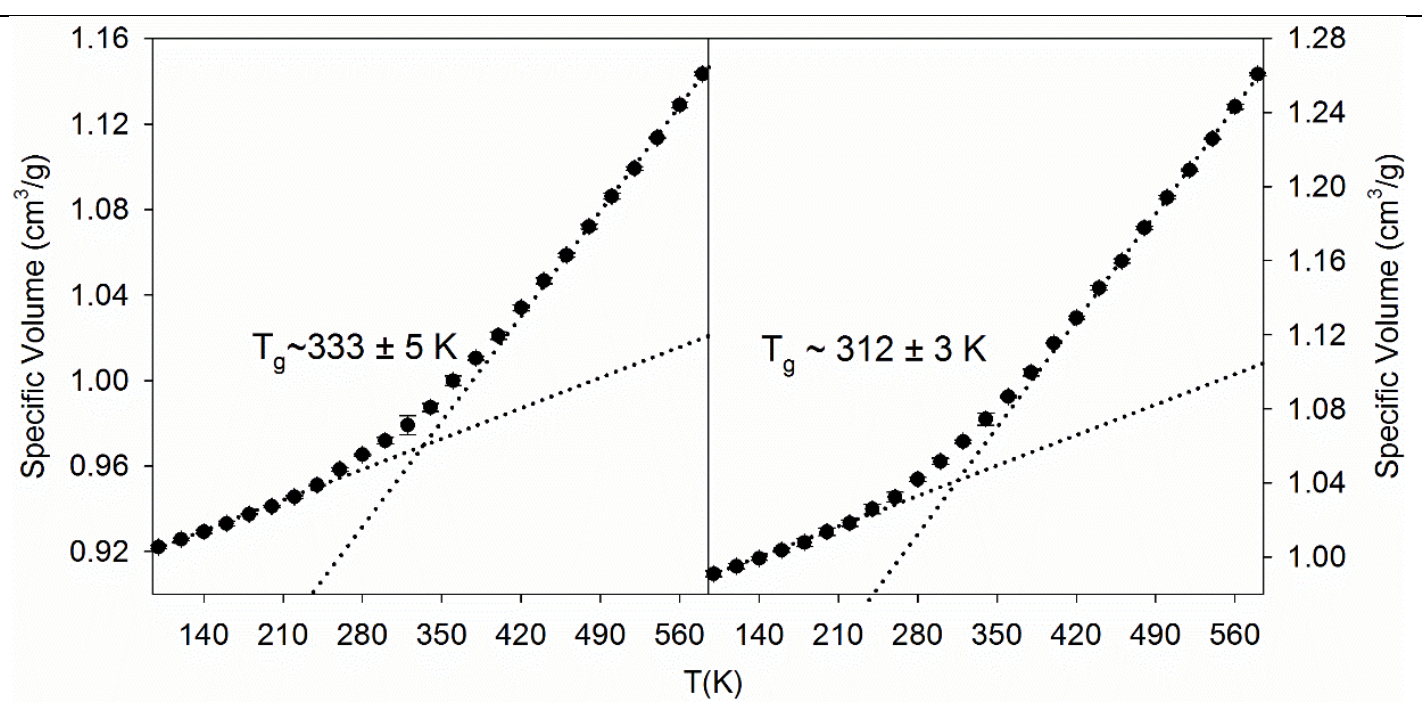

Figure 2. Specific volume vs. Temperature using atomistic modeling for calculation of $T_{g}$. (left) Nylon 6,6 and (right) Nylon 6,12 


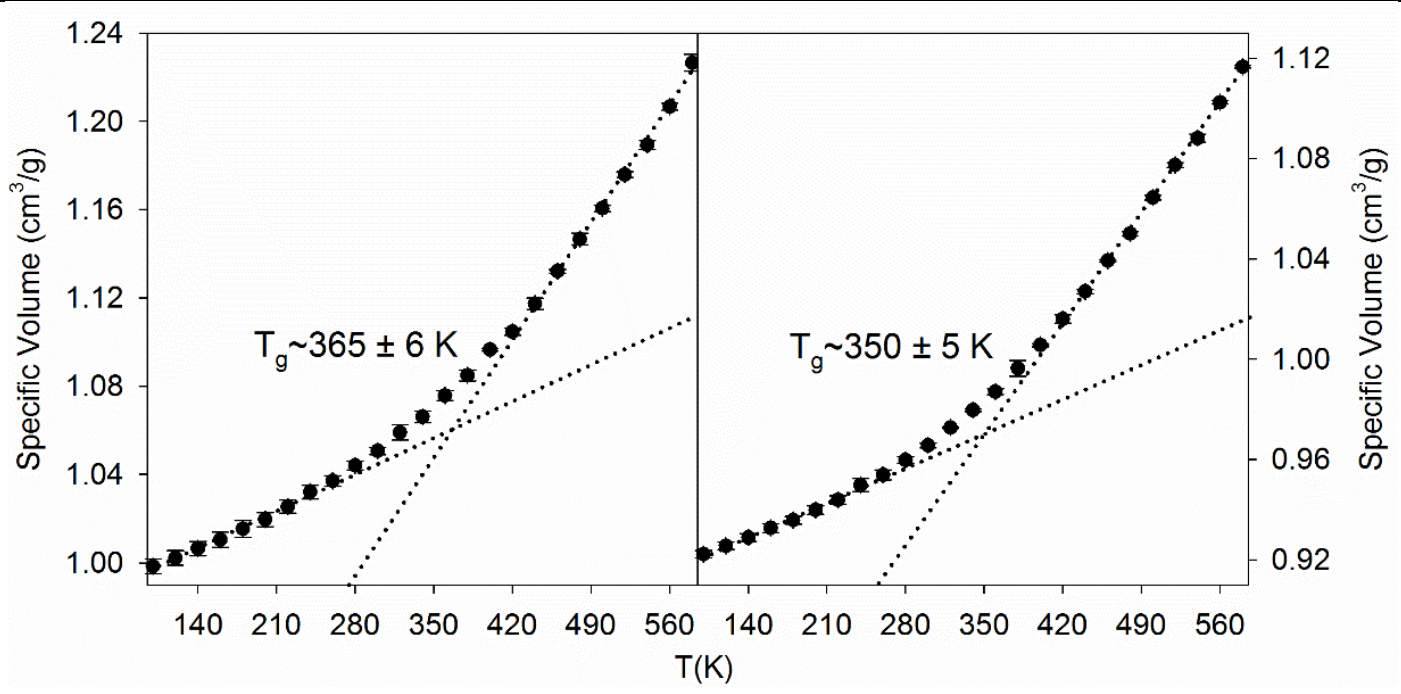

Figure 3. Specific volume vs. Temperature using atomistic modeling for calculation of $\boldsymbol{T}_{g}$. (left) Cycloaliphatic polyamide and (right) Aromatic Polyamide

Table 2. $T_{g}$ values from Atomistic Simulations

\begin{tabular}{|c|c|c|}
\hline Polymer & $\boldsymbol{T}_{\boldsymbol{g}}(\mathbf{K})$ from simulation & Experimental $\boldsymbol{T}_{\boldsymbol{g}}(\mathbf{K})$ \\
\hline Nylon 6,6 & $333 \pm 5$ & $323-341[35,40-42]$ \\
\hline Nylon 6,12 & $312 \pm 3$ & $319[43,44]$ \\
\hline Cycloaliphatic polyamide & $365 \pm 6$ & $413[39], 407[19]$ \\
\hline Aromatic Polyamide & $350 \pm 5$ & $383[19]$ \\
& & \\
\hline
\end{tabular}

From the simulation results, we observe that the $T_{g}$ of nylon 6,6 falls within the experimental range, while nylon 6,12 , and the cycloaliphatic polyamide follows the correct trend, though the values are slightly lower than those reported in the literature[35, 40-44]. This lower trend is expected since crystalline regions tend to lower the free volume exhibited by the material, the $T_{g}$ of semicrystalline materials tend to be higher than those exhibited by their purely amorphous analogs[2]. Even so, restrictions in accessible time scale in atomistic simulations results in the use of a much faster cooling rate, which would results in higher values of $T_{g}$ than 
experiment.[45] For nylon 6,6, better agreement was observed between the experimental and simulated values, where effects from both the higher cooling rate and the high crystallinity (4060\%)[38] may have contributed to this outcome.

The cycloaliphatic groups in the polyamide backbone is more rigid than the aliphatic groups, where higher chain backbone rigidity is typically associated with a higher $T_{g}$ due to a decrease in segmental mobility[46-48]. It is noteworthy that the calculated $T_{g}$ of cycloaliphatic polyamide is higher than that of the corresponding aromatic polyamide analog, which corroborates well with the experimental observation[19]. This characteristic is rather interesting as one would have expected that the presence of bis-phenyl rings would make the chain backbone more rigid[47, 48], therefore reducing the segmental mobility resulting in a higher $T_{g}$. The expected relationship between backbone rigidity and $T_{g}$ was observed in soy-based polyurethanes, where those with aromatic diisocyanates had a higher $T_{g}$ than the aliphatic analog[47]. We will investigate the effect of cycloaliphatic vs. aromatic rings on chain rigidity by considering dihedral angle distributions associated with rotation around the $\mathrm{C}-\mathrm{C}$ bond that connects the ring to the $\mathrm{CH}_{2}$ group located between the two rings in Section IV C. In addition, unlike the soy-based polyurethanes, these polyamides have a higher propensity towards the formation of intermolecular hydrogen bonding. This characteristic associated with polyamides but not polyurethane, could also play an important role, where the molecular influence, cycloaliphatic 4,4'-methylene-bis(cyclohexylamine) vs. aromatic 4,4'- methylenedianiline, can affect the overall chain packing, which presumably has a strong effect on the $T_{g}$.

These simulation results indicate that the choice of cycloaliphatic 4,4'-methylenebis(cyclohexylamine) can give rise to polyamides with a desired $T_{\mathrm{g}}$, much higher than that of nylons yet close to the $T_{g}$ of polycarbonate, which is of interest to engineering thermoplastics 
applications. It should be reiterated that the $T_{g}$ calculated in this study is associated with a purely amorphous system. This estimation maybe more appropriate for our model cycloaliphatic polyamide, which exhibits only small crystallites that do not scatter visible light.[39] For nylon 6,6 and 6,12 , the regularity and flexibility of these aliphatic polyamides, along with strong hydrogen bonding, contributes to crystallization[49]. In addition, the presence of rigid, aromatic phenyl rings would facilitate better chain ordering leading to the formation of crystalline regions, which can presumably affect the $T_{g}$ of these polyamides. However, these effects would be difficult or impossible to capture in an atomistic model given the short time scales accessible. Instead, coarse-grained meso-scale models will be used to study the effect of crystallinity in the future.

\section{Dihedral free energy barriers}

The main difference between the select model cycloaliphatic and aromatic polyamides is the presence of either a bis-cyclohexyl ring and bis-phenyl ring moieties associated with the corresponding 4,4'-methylene-bis(cyclohexylamine) and 4,4'-methylenedianiline, respectively, where the aromatic rings exhibit a planar structure while the cycloaliphatic analog can exhibit a variety of conformations (chair, boat, twist). In addition, the methylene moieties between both the bis-cyclohexyl rings and bis-phenyl rings can also facilitate flexibility through rotation of the associated dual ring structures. To investigate the effect of the ring type (aromatic vs. cycloaliphatic), we calculate the free energy associated with rotation of the rings with respect to a single C-C (b-d and e-d) bond as shown in Figure 4. This will offer some indication of how the cyclohexyl ring moieties can affect the segmental mobility, and correspondingly their influence on the $T_{g}$ of cycloaliphatic polyamides, in comparison with the corresponding aromatic polyamide analogs. 


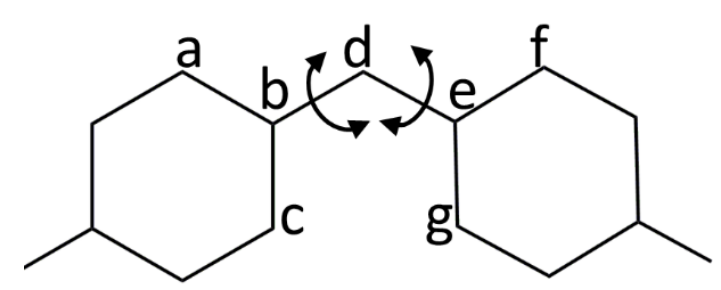

Figure 4. Atom labels associated with the calculation of the torsional angles in the cycloaliphatic and aromatic model polyamides. In this calculation, we consider rotation around two $\mathrm{C}-\mathrm{C}$ bonds noted by the arrows.

The free energy profile associated with the dihedral angle distribution is shown in Figure 5. For the aromatic polyamide, we observe the same symmetric distribution for the a-b-d-e, c-b-d-e, bd-e-f, and b-d-e-g dihedral angles (see Figure 4 for labeling), where the maximum energy barrier for rotation is $\sim 1 \mathrm{kcal} / \mathrm{mol}$. Conversely, the cycloaliphatic polyamide exhibits two different dihedral distributions depending on the relative location of hydrogen atom connected at the carbon atom at b or e, which are mirror images around $\psi=0^{\circ}$. In Figure 5, we only show one of the mirror image distributions. 


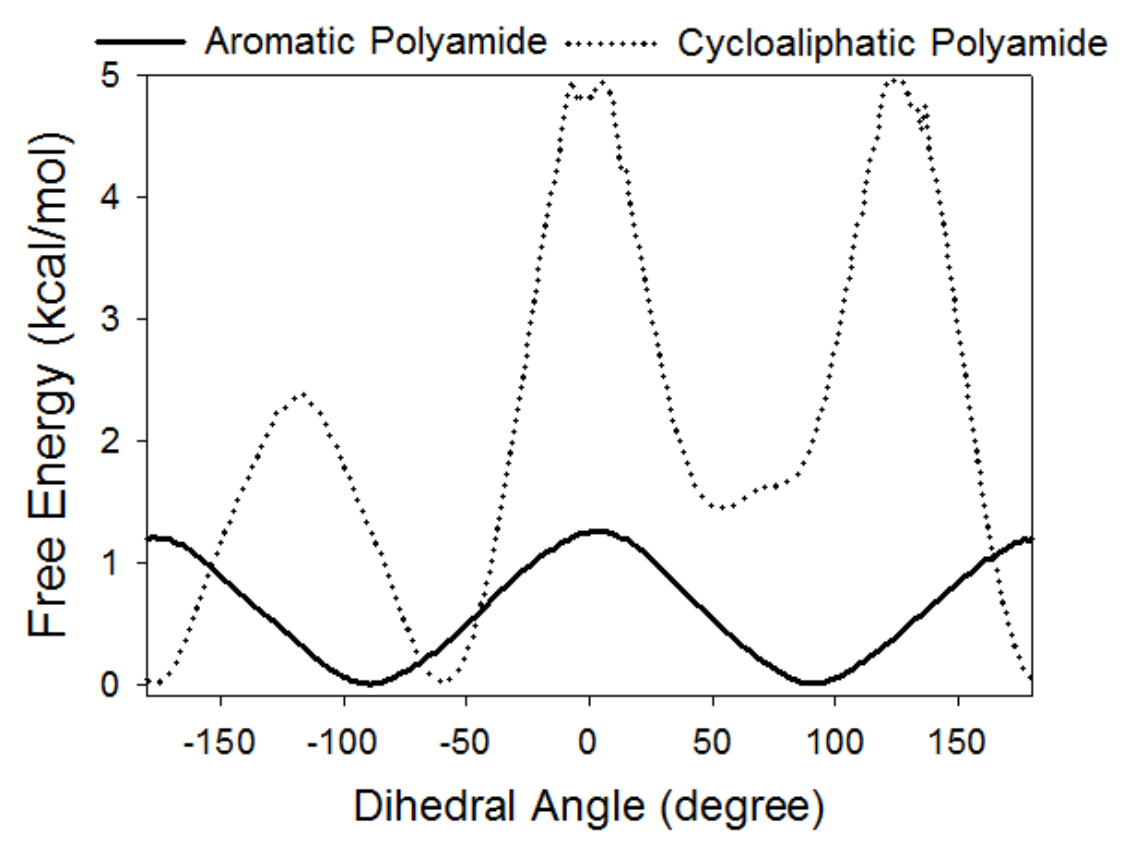

Figure 5. Free energy profile as a function of dihedral angles for aromatic polyamide and a representative distribution for cycloaliphatic polyamide.

The lack of symmetry in the free energy profile around $\psi=0^{\circ}$ observed in the

cycloaliphatic polyamide can be explained visually through a Newman projection along the b-d or e-d bonds. This is shown for the b-d bond in Figure 6, where the conformations are listed as a function of the dihedral angle associated with the energy profile shown in Figure 5. At $\psi=-120^{\circ}$, the energy barrier is calculated to be $\sim 2 \mathrm{kcal} / \mathrm{mol}$, where the conformation has each carbon eclipsing a hydrogen. The highest energy conformation occurs at $\psi=0^{\circ}$ and $120^{\circ}$, where two carbon atoms eclipse each other and one carbon eclipses a hydrogen. The lowest energy conformations are staggered, and have two carbons in the gauche position and two carbons in the anti-position. 


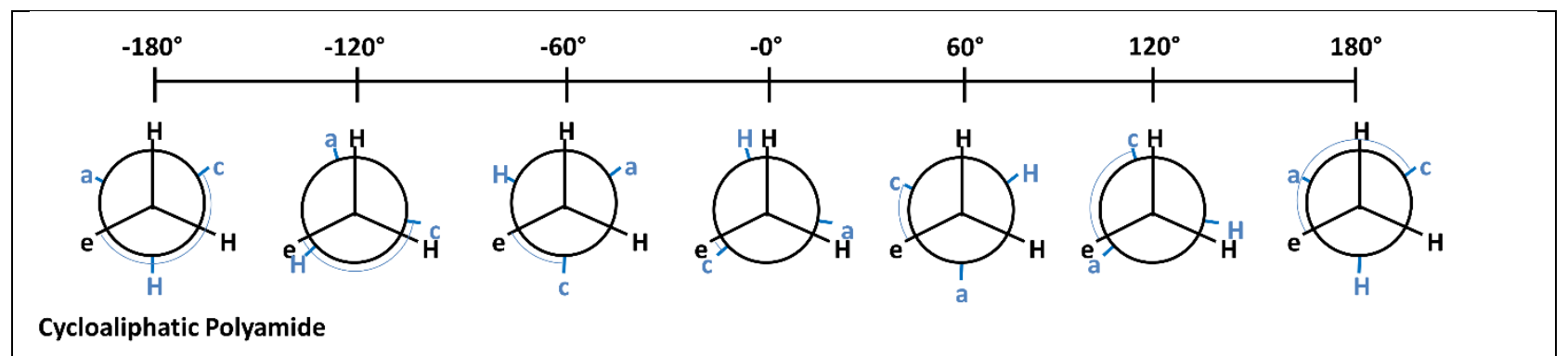

Figure 6. Newman potential along the b-d bond for cycloaliphatic polyamide. Overall, the cycloaliphatic polyamide exhibits larger rotational barriers around the b-d and e-d bonds than the aromatic analogue. The higher rotational barrier exhibited by the cycloaliphatic polyamide indicates that torsion rotation around this bond is more constrained, reducing the overall mobility. This chain rigidity can account for the higher $T_{g}$ observed in the cycloaliphatic polyamide.

D. Intermolecular Hydrogen Bonding

The $T_{g}$ is also affected by differences in the molecular packing which can be attributed in part to differences in intermolecular hydrogen bonding, since a denser molecular packing leads to a lower free volume[50-52]. The number and stability of these hydrogen bonds can also give us an idea of their effect on chain mobility which could lead to large changes in $T_{g \text {.[53] }}$ To determine the propensity of these model polyamides towards the formation of intermolecular hydrogen bonding for our four systems, we calculated both the average number and lifetime of these hydrogen bonds at ambient conditions. Since the total number of amide hydrogen bonding pairs in all four systems are the same, absolute numbers of H-bonds can be compared.

\begin{tabular}{|} 
Table 3. Hydrogen Bonding values from Atomistic Simulations \\
\begin{tabular}{|c|c|c|}
\hline Polymer & Avg \# of H-bonds at 298K & $\begin{array}{c}\text { Avg lifetime of H- } \\
\text { bond (ps) }\end{array}$ \\
\hline Nylon 6,6 & $440.36 \pm 8.24$ & $6.98 \pm 0.02$ \\
\hline
\end{tabular}
\end{tabular}




\begin{tabular}{|l|l|l|l|}
\hline & Nylon 6,12 & $424.60 \pm 8.29$ & $7.35 \pm 0.15$ \\
\cline { 2 - 4 } $\begin{array}{l}\text { Cycloaliphatic } \\
\text { polyamide }\end{array}$ & $371.73 \pm 6.68$ & $8.81 \pm 0.13$ \\
\cline { 2 - 4 } Aromatic Polyamide & $391.28 \pm 8.23$ & $6.33 \pm 0.29$ \\
\hline
\end{tabular}

Results are listed in Table 3, where we observe that nylon 6,6 is the most prone to hydrogen bonding, while the cycloaliphatic polyamide is the least. This is expected since polymers with smaller repeat units tend to have higher hydrogen bond densities. This is particularly true when comparing nylon 6,6 and 6,12 , where both polyamides exhibit a similar number of hydrogen bonds, but the smaller repeat unit of nylon 6,6 make it so that the hydrogen bonds occupy a smaller volume resulting in a higher hydrogen bonding density (nylon 6,6: $4.01 \pm 0.005 / \mathrm{nm}^{3}$ vs. nylon $\left.6,12: 2.61 \pm 0.002 / \mathrm{nm}^{3}\right)$. Also, bulky rings such as those in both the cycloaliphatic polyamide and aromatic polyamide can hinder the formation of hydrogen bonds. Since the van der Waals volume, which characterizes the space that is occupied by the molecule and is impenetrable to other molecules under normal thermal conditions, is larger for the cycloaliphatic $\operatorname{ring}\left(53.3 \mathrm{~cm}^{3} / \mathrm{mol}\right)$ than the aromatic $\operatorname{ring}\left(43.3 \mathrm{~cm}^{3} / \mathrm{mol}\right)$, the cycloaliphatic group is bulkier[27]. Consequently, the cycloaliphatic polyamide has a lower number of hydrogen bonds and hydrogen bonding density (cycloaliphatic: $1.75 \pm 0.004 / \mathrm{nm}^{3}$ vs. aromatic: $1.97 \pm 0.003 / \mathrm{nm}^{3}$ ). Furthermore, structural conformations associated with the cyclohexyl group in a cycloaliphatic polyamide may also have a strong influence on its propensity towards intermolecular hydrogen bonding. This will be further evaluated and reported in a future publication. 

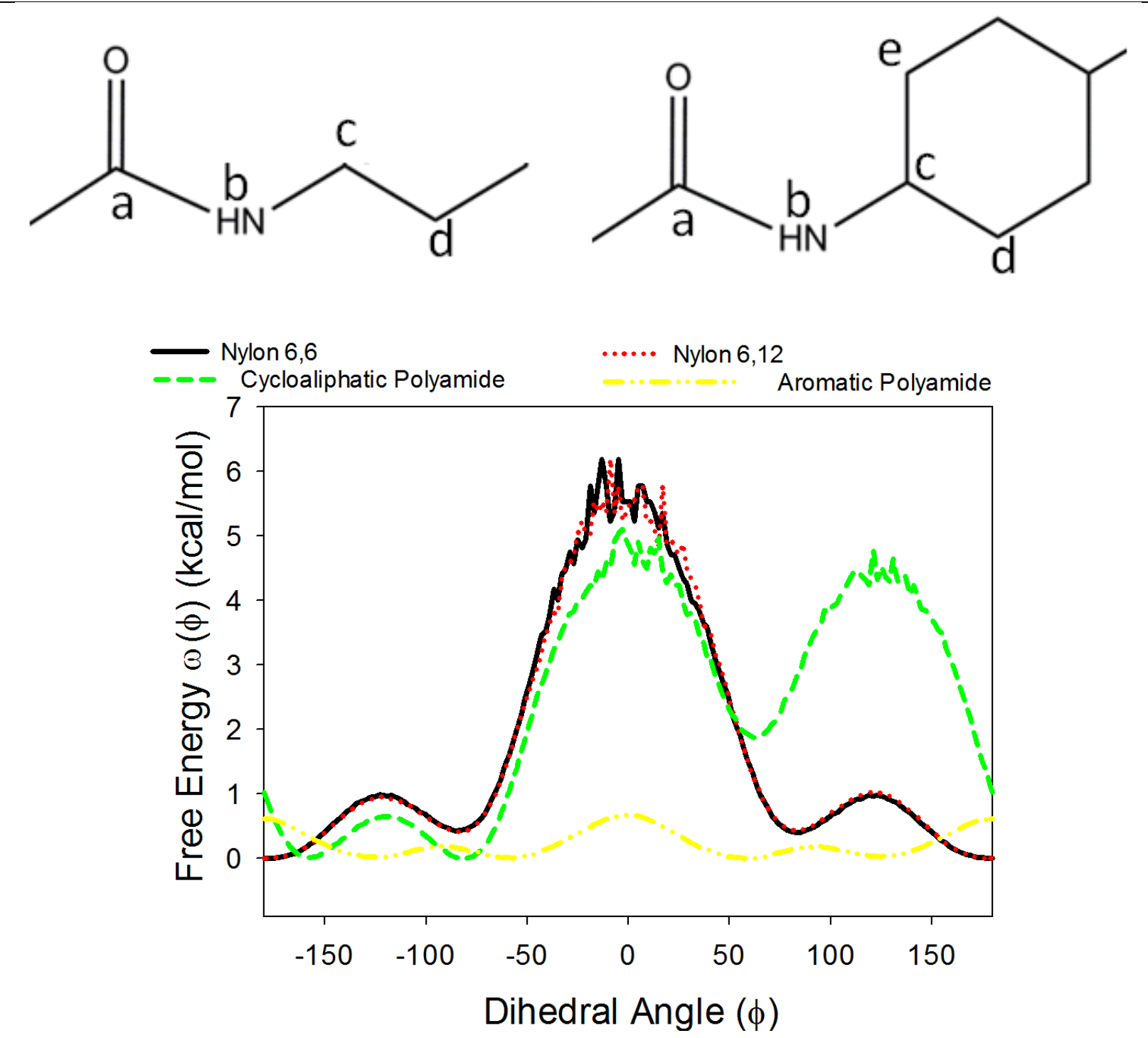

Figure 7. (Top) Atoms used in the dihedral angle calculation in the model polyamides (shown here for the (left) aliphatic and (right) cycloaliphatic polyamide). (Bottom) Dihedral angle of the polymer backbone containing the amide group.

From our molecular simulations, we observed that the cycloaliphatic polyamide exhibits the longest average lifetime of hydrogen bonding while the aromatic polyamide has the shortest average lifetime. This indicates more persistent hydrogen bonding interaction in the cycloaliphatic polyamide. On the other hand, the aromatic polyamide has the shortest average lifetime since more rigid structures such as the aromatic ring can make it harder to recover from 
disrupted hydrogen bonding. These simulation results also suggest that intermolecular hydrogen bonding appears to be much more robust in cycloaliphatic polyamide than the corresponding aromatic analog, despite the better packing afforded through the aromatic ring configuration.

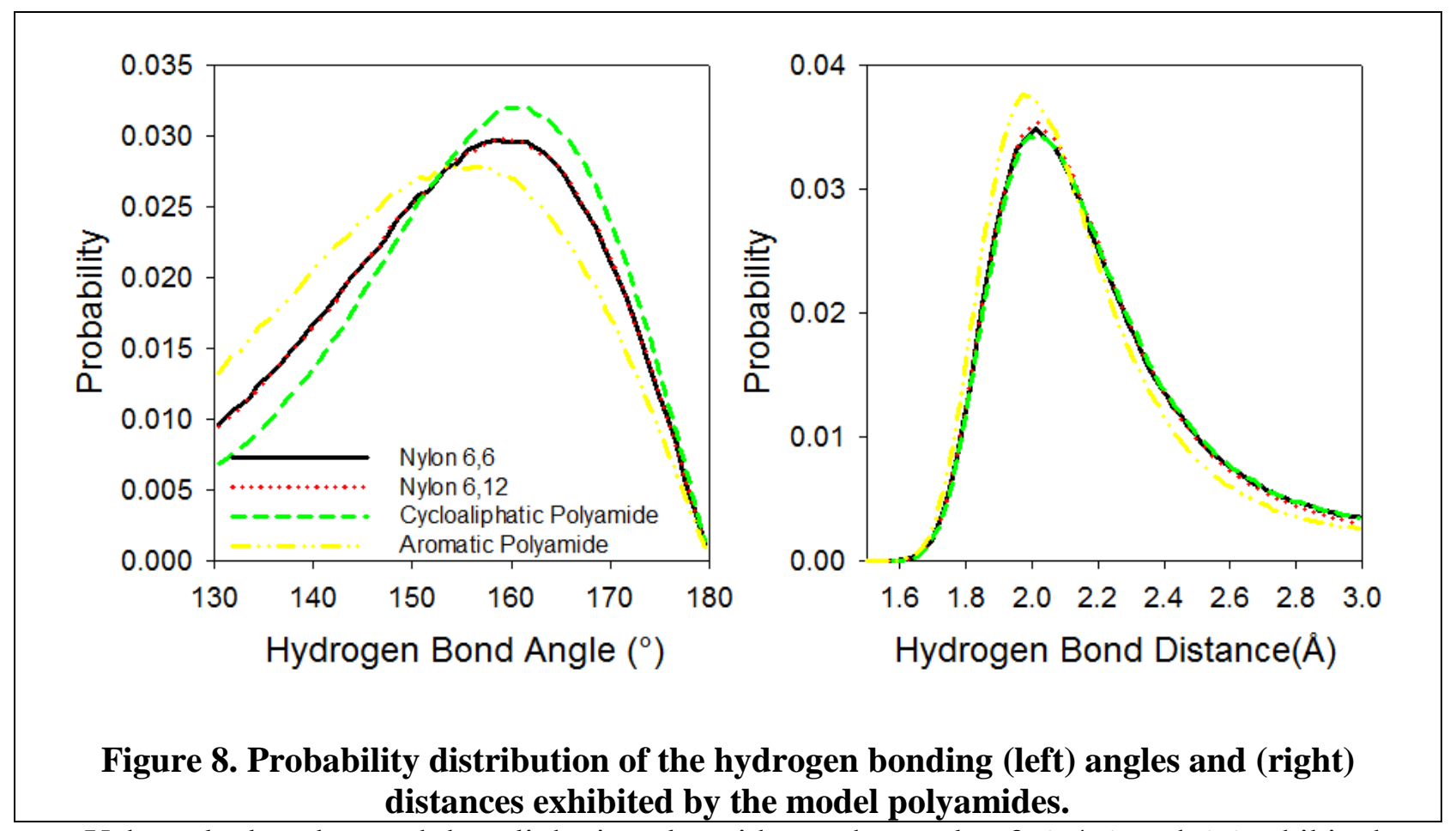

Yeh et al. also observed that aliphatic polyamides such as nylon 3,6, 4,6, and 6,6 exhibited a larger number of hydrogen bonds than their furan polyamides counterparts at room temperature, yet these aromatic ring based polymers exhibited a lower hydrogen bonding lifetime. This observation agrees with our results when we compare nylon 6,6, 6,12 and our model aromatic polyamide, but the cycloaliphatic polyamide exhibits the longest average hydrogen bond lifetime. To further investigate causes for this longer lifetime, we calculated the dihedral angle associated with the polymer backbone which contains the amide group for our model polyamide systems. We use the same procedure outlined in Section IV c, on the a-b-c-d and a-b-c-e atoms shown in Figure 7 for the aromatic and cycloaliphatic polyamides and a-b-c-d for nylon 6,6 and 6,12. The free energy profile as a function of the dihedral angle is shown in Figure 7. The plot indicates that the energy barrier to rotation (less than $1 \mathrm{kcal} / \mathrm{mol}$ ) is much lower for the aromatic 
polyamide, making it the most mobile when compared to the other model polyamides. The free energy profile for cycloaliphatic polyamide indicates a more constrained motion than both aliphatic polyamides, which exhibits a similar free energy profile. Although the aliphatic polyamides exhibit a large energy barrier $(\sim 6 \mathrm{kcal} / \mathrm{mol})$ when the dihedral angle is $\sim 0^{\circ}$, the energy barriers we observe at $\sim \pm 120^{\circ}$ are much smaller $(\sim 1 \mathrm{kcal} / \mathrm{mol})$. As in Section IV C, the cycloaliphatic polyamide exhibits two different dihedral distributions depending on the relative location of hydrogen atom, which are mirror images around $\phi=0^{\circ}$. Thus, the shortest hydrogen bond lifetime observed for the aromatic polyamide is due to the low energy barriers to rotation, while the longest lifetime detected in cycloaliphatic polyamide is due to a more constrained energy landscape. In aromatic polyamides, the shorter hydrogen bonding lifetime can be associated with the mobility of the backbone near the amide group. We observed a similar trend in the energy barriers when we considered the dihedral angles between the phenyl or cyclohexyl rings in Section IV c. This calculation does not explain why the hydrogen bonding lifetime of nylon 6,6 is different than 6,12 given their similar free energy distribution. This could be due to statistical errors where greater sampling and replicas may be needed.

Although a hydrogen bond is considered to be formed based on an angle and distance criteria, we will also consider their distribution. As shown in Figure 8, the distribution of hydrogen bond distances is very similar for all of the model polyamides, though differences were observed in the angles. The aromatic polyamide exhibits a broad distribution, while the other polyamides exhibit a more narrow distribution centered around $160^{\circ}$. Since the closer the $\angle \mathrm{N}$ $\mathrm{H}$... O angle is to being linear $\left(180^{\circ}\right)$, the stronger the hydrogen bond is, the distribution shown in Figure 8, suggests that the cycloaliphatic polyamide has a highest propensity for strong hydrogen bonds, while the aromatic polyamides exhibits the lowest. Since the angle distribution 
for the aromatic polyamide is broader, there is a higher probability for smaller angles than the other polyamides. This may make it more sensitive to the mobility of the connecting polymer backbone which can also contribute to the shorter hydrogen bonding lifetime.

The average number and lifetime of intermolecular hydrogen bonds as a function of temperature were also calculated and are shown in Figure 9. As expected, as temperature increases the segmental mobility increases, which would result in a weakening of the intermolecular hydrogen bonding interaction. Furthermore, it is noteworthy that the temperature profile of the average lifetime of cycloaliphatic polyamide is very similar to those of linear nylons, which is significantly different from that of aromatic polyamide. This may indicate that the segmental dynamics of cycloaliphatic polyamide is much closer to the mobility of aliphatic nylons, which may be critical when considering the influence of segmental dynamics in the dynamic mechanical deformation response. Furthermore, the difference in the average lifetime between our model polyamides increases towards lower temperature, indicating that the cycloaliphatic polyamide can potentially have better dynamic mechanical strength at high strain rates than the corresponding aromatic polyamide analogs. These observations are critical particularly when considering the design of polyamides with improved dynamic mechanical performance.
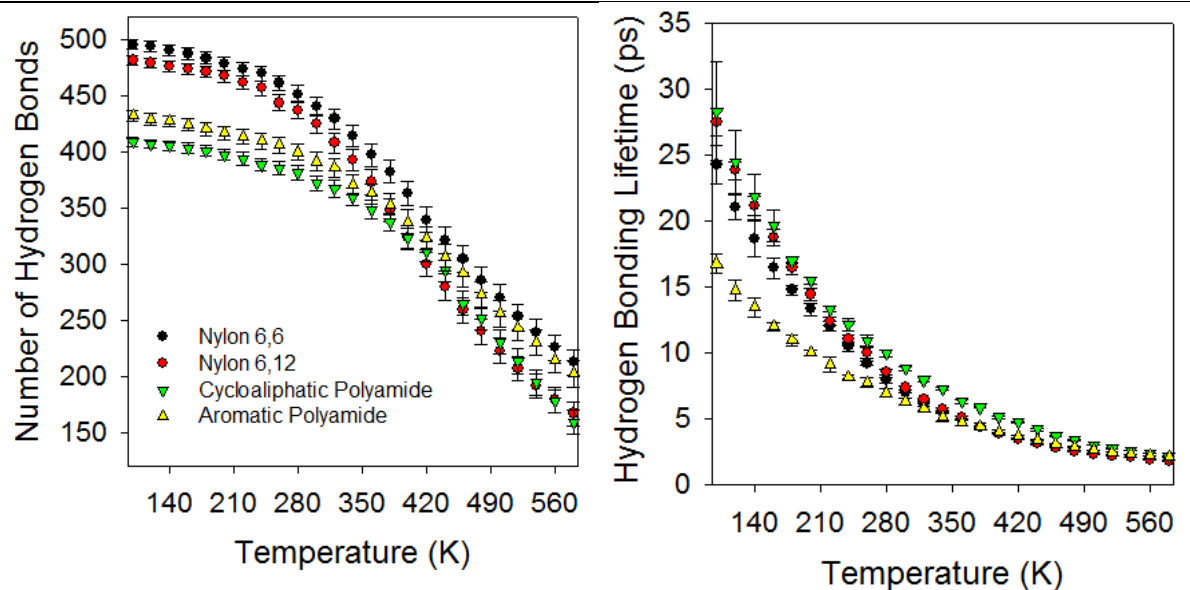


\section{Figure 9. Average (left) number and (right) lifetime of hydrogen bonds as a function of temperature.}

The dependence of intermolecular hydrogen bonding on temperature has been investigated experimentally on polymers through techniques such as infrared spectroscopy. For polyurethane block polymers, it was observed that the extent of hydrogen bonding exhibited in the hard segments are influenced by the $T_{g}$ of the hard segment[54]. Paik Sung and Schneider[55] also studied the temperature effect on hydrogen bonding in toluene diisocyanate based polyurethanes, where they found that the influence of temperature on the extent of hydrogen bonding is independent of structural organization or morphology of the hard and soft segment. In both studies, a discontinuity in intermolecular hydrogen bonding was noted at a temperature similar to the $T_{g}$ of the hard segments. It has been suggested that the incipient of the $T_{g}$ marks the onset of dissociation of hydrogen bonding since the loss of segmental mobility at $T_{g}$ can result in the immobilization of hydrogen bonds $[55,56]$. To determine the influence of hydrogen bond dissociation on $T_{g}$, we calculated the dissociation temperature using the same protocol that was used to calculate the $T_{g}$. For consistency we used the same temperature points to calculate the linear fits in the rubbery and glassy regime. Extracted values of the onset temperature for hydrogen bond dissociation are shown in Table 4. By comparing Table 2 and Table 4, it is clear that same trend occurs in both the dissociation temperature and the $T_{g}$ for our polyamide systems. In addition, it is important to note that the dissociation temperatures are consistency lower than $T_{g}$ for all of the model polyamides, but the difference is much smaller for both the cycloaliphatic and aromatic polyamide systems. This could suggest that hydrogen bonding in the amorphous phase plays a larger role in $T_{g}$ for the cycloaliphatic and aromatic polyamide systems. 


\begin{tabular}{|} 
Table 4. Onset temperature for hydrogen bond dissociation \\
$\qquad$\begin{tabular}{|c|c|}
\hline Polymer & $\begin{array}{c}\text { Temperature at the } \\
\text { discontinuity in the \# of } \\
\text { hydrogen bonds (K) }\end{array}$ \\
\hline Nylon 6,6 & $218.28 \pm 26.98$ \\
\hline Nylon 6,12 & $156.88 \pm 5.49$ \\
\hline Cycloaliphatic polyamide & $333.61 \pm 18.72$ \\
\hline Aromatic Polyamide & $287.68 \pm 22.71$ \\
\hline
\end{tabular}
\end{tabular}

E. Fractional and Cavity Free Volume

Free volume, or unoccupied empty space, in a material occurs due to inefficient packing of the polymer chains. This empty space enables the material to undergo segmental motion such as rotation and translation. The FCV calculated at ambient conditions through the CESA method follow the same trend as the fractional free volume calculated through the Bondi method when the density was taken from simulation (see Appendix A). More specifically, the calculated FCV decreases in the following order: cycloaliphatic polyamide $(0.0847 \pm 0.001)>$ nylon 6,12 $(0.0792 \pm 0.001)>$ aromatic polyamide $(0.0765 \pm 0.001)>$ nylon $6,6(0.0758 \pm 0.001)$. Again, this calculation does not take into account of the free volume attributed to any of the crystalline regions, though the amorphous phase tends to have the largest proportion of free volume. To further characterize how the FCV is distributed, cluster properties such as volume, surface area, span, and radius of gyration were calculated, where weighted distributions are shown in Figure 

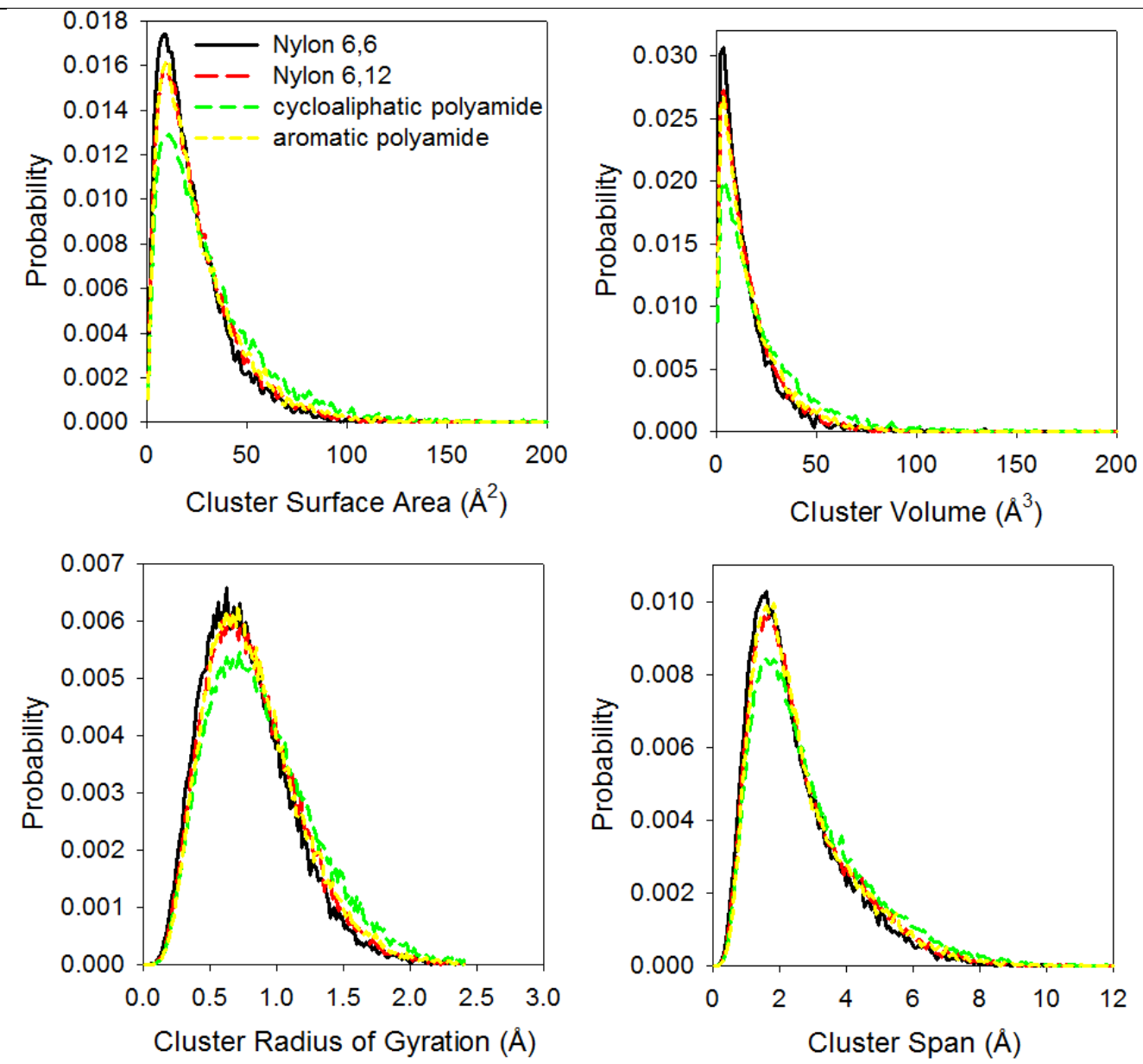

Figure 10. Cluster properties such as surface area, volume, radius of gyration, and span associated with the free volume under ambient conditions.

10 and average values are given in Table 5. The cycloaliphatic polyamide exhibits the broadest

distribution in the cluster surface area, volume, span and radius of gyration, while nylon 6,6 has

the most narrow distribution. The average values shown in Table 5 reveal the following trend for all of the shape parameters considered: cycloaliphatic polyamide $>$ aromatic polyamide $>$ nylon $6,12>$ nylon 6,6, where both nylon 6,12 and the aromatic polyamide exhibit very similar average values and distributions. The trend that we observe in the shape parameters do not agree with the trend in the FCV calculations where we observed a higher value in nylon 6,12 than the aromatic polyamide. This is because the FCV measures the amount of the cavity free volume, but not how the free volume is distributed. 
Fox and Flory's theory[57] of the $T_{g}$ suggests that during the cooling process the free volume of a polymer experiences a sudden change at the glass-transition temperature. This can manifest in a discontinuity at $T_{g}$, a feature which has been demonstrated in numerous molecular dynamics $[5,58]$ and experimental[58-61] studies. Experimentally this free volume can be measured using PALS $[58,59,62]$ which have been shown to agree with FCV calculations through CESA. To determine if this characteristic is also apparent among these model polyamides, values

\begin{tabular}{|c|c|c|c|c|}
\hline & $\begin{array}{l}\text { Cluster Surface } \\
\text { Area }\left(\AA^{2}\right)\end{array}$ & $\begin{array}{l}\text { Cluster Volume } \\
\left(\AA^{3}\right)\end{array}$ & $\operatorname{Span}(\AA)$ & $\begin{array}{l}\text { Radius of } \\
\text { Gyration }(\AA)\end{array}$ \\
\hline \begin{tabular}{|l} 
Nylon $\mathbf{6 , 6}$ \\
\end{tabular} & $10.61 \pm 0.076$ & $3.80 \pm 0.043$ & $1.78 \pm 0.012$ & $0.63 \pm 0.004$ \\
\hline \begin{tabular}{|l} 
Nylon 6,12 \\
\end{tabular} & $11.76 \pm 0.11$ & $4.41 \pm 0.060$ & $1.89 \pm 0.010$ & $0.66 \pm 0.003$ \\
\hline $\begin{array}{l}\text { Cycloaliphatic } \\
\text { polyamide }\end{array}$ & $13.66 \pm 0.13$ & $5.69 \pm 0.074$ & $2.02 \pm 0.010$ & $0.71 \pm 0.003$ \\
\hline $\begin{array}{l}\text { Aromatic } \\
\text { polyamide }\end{array}$ & $12.19 \pm 0.039$ & $4.66 \pm 0.009$ & $1.92 \pm 0.005$ & $0.68 \pm 0.001$ \\
\hline
\end{tabular}

of $T_{g}$ were extracted from a plot of FCV vs. temperature (see Figure 11). Calculated $T_{g}$ values from the FCV are shown in Table 6, where we observe the same trend in the $T_{g}$ shown in Table 2. In addition, although the standard deviation is much larger, the values of $T_{g}$ extracted through the FCV fall within the range of values calculated from the specific volume and temperature for all the polyamides except of nylon 6,12 . This could be due to an insufficient number of snapshots that were analyzed due to the high computational cost. Even so, the trend suggests that the FCV, as calculated through CESA, is a quantity that affects the $T_{g}$ in these materials. 


\begin{tabular}{|l} 
Table 6. Values of $\boldsymbol{T}_{g}$ extracted from FCV vs. temperature data. \\
$\qquad$\begin{tabular}{|c|c|}
\hline Polymer & $\begin{array}{c}\text { Temperature at the } \\
\text { discontinuity in FCV (K) }\end{array}$ \\
\hline Nylon 6,6 & $312 \pm 19$ \\
\hline Nylon 6,12 & $276 \pm 20$ \\
\hline Cycloaliphatic polyamide & $347 \pm 23$ \\
\hline Aromatic Polyamide & $338 \pm 10$ \\
\hline
\end{tabular}
\end{tabular}

Large scale motion of the polymers depends not only on the overall FCV but on its

distribution in the material, since the free space must be large enough to accommodate local chain rearrangement. Experimentally, the size of free volume cavities has been quantified through PALS using semi-empirical equations such as the Tao-Eldrup (TE) equation which assumes that the free volume hole size is spherical with an effective radius. This method has been used to estimate the free-volume of voids in numerous polymers[60, 61]. Plots of the mean

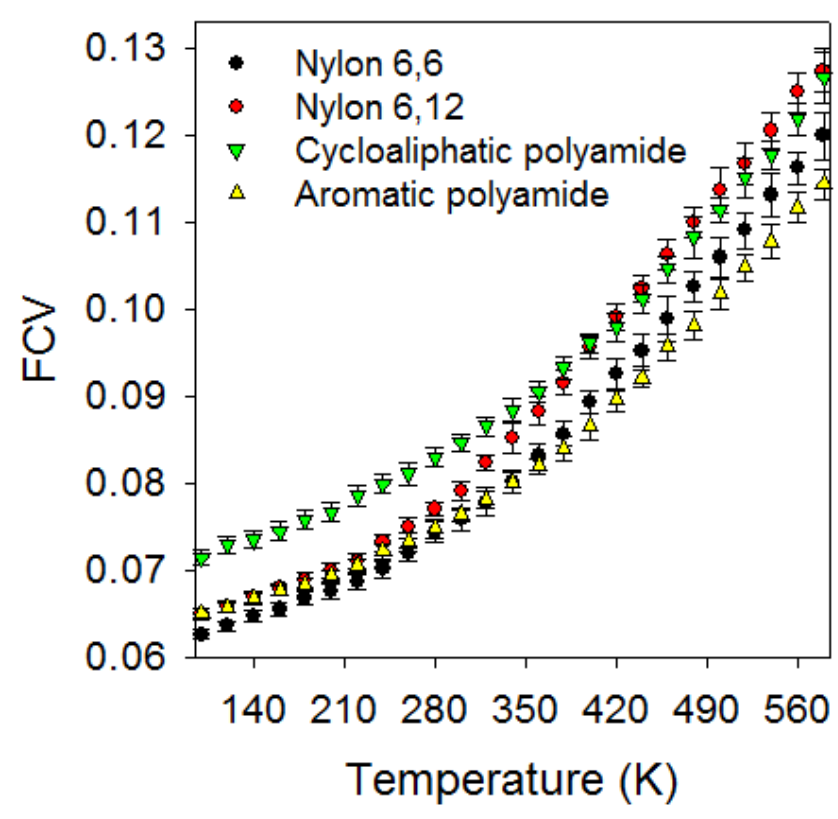

Figure 11. Fractional Cavity Volume as a function of temperature.

hole size as a function of temperature have also been used to extract values of $T_{g}$, where values 
extracted have been shown to be similar to those obtained using other experimental methods
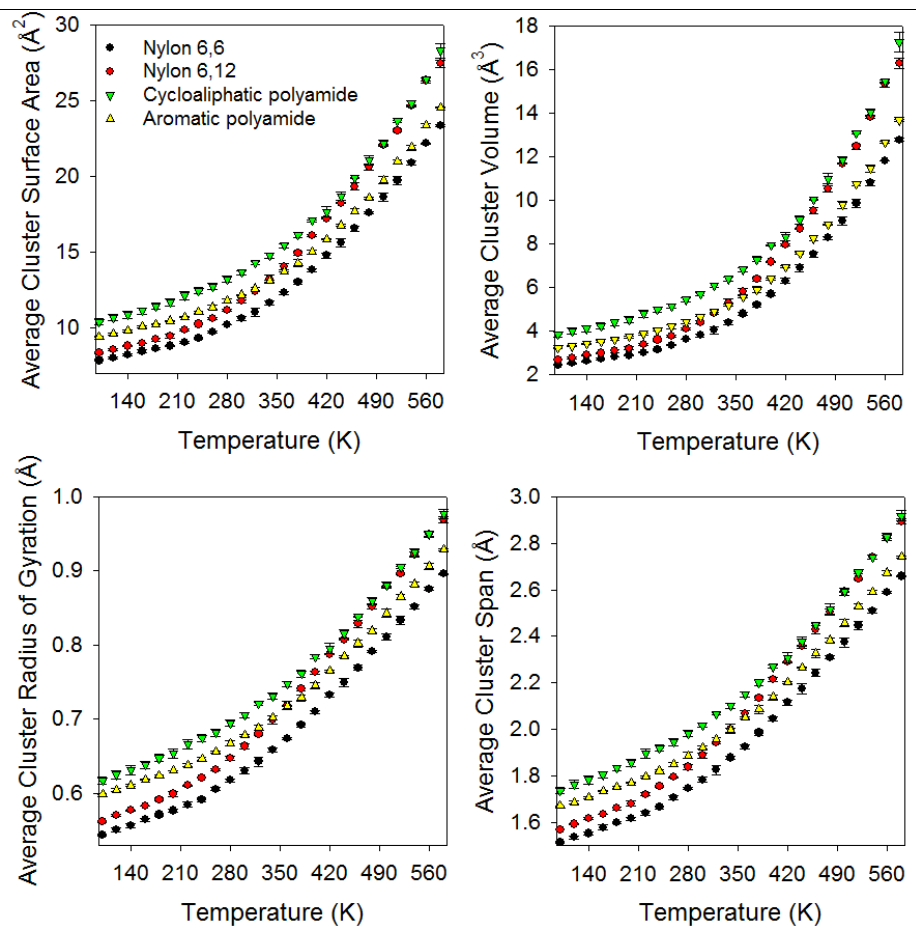

Figure 12. Average values of cluster shape parameters as a function of temperature.

such as differential scanning calorimetry.

Computationally, we can take this a step further and characterize the shape of the clusters, beyond the simple spherical approximation typically used experimentally. Average values of the cluster shape parameters (surface area, volume, radius of gyration, and span) are plotted as a function of temperature in Figure 12. At low temperatures, all of the cluster shape parameters follow the same trend we observed at ambient conditions: nylon $6,6<$ nylon $6,12<$ aromatic polyamide < cycloaliphatic polyamide. As in the FCV, the shape parameters for nylon 6,12 starts to increases faster than the other polyamides, where it surpasses the model aromatic polyamide in terms of size at $\sim 340 \mathrm{~K}$. At higher temperatures (up to $580 \mathrm{~K}$ ) it exhibits similar amounts of free volume as the cycloaliphatic polyamide. 
Similarly to the FCV, these contributions may also exhibit a transition at $T_{g}$. To determine if the $T_{g}$ is discernible through the shape parameters, the same procedure described previously was used to extract temperature values associated with the discontinuity. From Table 7, we show that the average values exhibit the same trend as the $T_{g}$ values given in Table 2. Even so, the cluster surface area, volume, and radius of gyration are statistically equivalent between the two aliphatic polyamides, and between the cycloaliphatic and aromatic polyamides. Although the cluster span is similar for the cycloaliphatic and aromatic polyamides, nylon 6,6 is statistically smaller than nylon 6,12. Even though this study suggests a correlation between these shape parameters and the $T_{g}$, a more comprehensive study needs to be performed.

Table 7. Values of $T_{g}$ extracted from plots of the shape parameter vs. temperature.

\begin{tabular}{|l|l|l|l|l|}
\hline & \multicolumn{4}{|c|}{ Temperature (K) at the discontinuity in cluster } \\
\hline Polymer & Surface area & Volume & $\begin{array}{l}\text { Radius of } \\
\text { Gyration }\end{array}$ & Span \\
\hline Nylon 6,6 & $356 \pm 5$ & $382 \pm 5$ & $312 \pm 8$ & $318 \pm 5$ \\
\hline Nylon 6,12 & $342 \pm 10$ & $372 \pm 8$ & $291 \pm 13$ & $298 \pm 12$ \\
\hline $\begin{array}{l}\text { Cycloaliphatic } \\
\text { polyamide }\end{array}$ & $383 \pm 14$ & $401 \pm 9$ & $351 \pm 17$ & $361 \pm 22$ \\
\hline $\begin{array}{l}\text { Aromatic } \\
\text { polyamide }\end{array}$ & $375 \pm 8$ & $394 \pm 3$ & $347 \pm 19$ & $345 \pm 14$ \\
\hline
\end{tabular}

\section{Conclusions}

The $T_{g}$ is shown to be dependent on molecular properties such as the free volume and the conformational mobility associated with the polymer, both of which are affected by the extent of the hydrogen bonding interaction. Using atomistic molecular dynamics simulations, we calculated the $T_{g}$ for four model polyamide systems: nylon 6,6 , nylon 6,12 , and a select model cycloaliphatic and its corresponding aromatic polyamide analog. The $T_{g}$ was extracted from a plot of specific volume vs. temperature, following the traditional method utilized in molecular dynamics simulations. Although our model only considered the amorphous phase, it was able to 
capture the correct trend in the $T_{g}$ : cycloaliphatic polyamide $(365 \mathrm{~K} \pm 6 \mathrm{~K})>$ aromatic polyamide $(350 \mathrm{~K} \pm 5 \mathrm{~K})>$ nylon $6,6(333 \mathrm{~K} \pm 5 \mathrm{~K})>$ nylon $6,12(312 \mathrm{~K} \pm 3 \mathrm{~K})$.

Typically, both the chain rigidity and the extent of free volume can influence on the $T_{g}$ of polymers. Both the cycloaliphatic and aromatic polyamides are more rigid than the aliphatic polyamides due to the presence of ring structures along the polymer backbone. To further elucidate the influence of the bis-cyclohexyl moieties vs. bis-phenyl rings in these two model polyamides, we characterized the dihedral free energy barriers associated with rotation of the dual ring moieties for both systems to give an indication of the local structural rigidity. The calculated energy barrier for rotation is higher for the cycloaliphatic analogue, indicating a more constrained torsion angle, making it less mobile.

Hydrogen bonding can affect the chain rigidity and free volume by affecting the molecular packing. The effect of hydrogen bonds on chain rigidity is beyond the scope of this paper but may be studied in a future publication by considering changes in free energy barriers associated with rotation when the hydrogen bonding interactions are excluded. At ambient conditions, the cycloaliphatic polyamide exhibits less mobility in the polymer backbone around the amide group and the hydrogen bonds that are formed are stronger than the other model polyamides, which contributes to its longer hydrogen bonding lifetime. For the range of temperatures considered (100-580K), nylon 6,6 exhibits a consistently higher number of hydrogen bonds than the other polyamides, which promotes the distribution of free volume towards smaller clusters. Although nylon 6,12, exhibits a similar trend as nylon 6,6, it exhibits a sharp decrease in the number of hydrogen bonds at high temperatures. At low temperatures the number of hydrogen bonds for nylon 6,12 is higher than the cycloaliphatic and aromatic polyamide, but at high temperatures the average number is lower. This same trend in the number of hydrogen bond is also exhibited in 
the free volume, where larger free volume clusters are seen at high temperature than those exhibited in nylon 6,6. Similar trends are observed with our model cycloaliphatic and aromatic polyamides, where the former has a larger amount of free volume than the latter, and where we observe a near parallel trend for the entire range of temperatures considered. This trend persists in the overall fractional cavity volume and the cluster shape parameters. Thus there is a clear association between the number of hydrogen bonds and the extent of free volume. In addition, the effect of temperature on the hydrogen bonding lifetime was also explored, where differences are apparent at lower temperatures yet begin to disappear at higher temperatures.

Properties such as the free volume exhibit an abrupt change at the $T_{g}$. This feature allows for the $T_{g}$ to also be extracted from the temperature profile of fractional free volume. Average values of the $T_{g}$ 's extracted through this plot follow the correct trend observed through the density vs. temperature plot, but the error bars tend to be larger, which may be due to insufficient sampling of snapshots due to the high computational cost associated with these calculations. The same can be said when the $T_{g}$ was extracted from the cluster shape parameters. As mentioned previously, there is a relationship between the number of hydrogen bonds and the free volume, where we also observed an abrupt change at or around the $T_{g}$. Extracted values of the $T_{g}$ from these plots, also agree well with those obtained using the traditional method, though the value for nylon 6,12 was slightly lower.

Overall, we have demonstrated a comprehensive study on the $T_{g}$ for our model polyamide systems, by considering factors such as chain rigidity, free volume, and hydrogen bonding. Nylon 6,6 has lower free volume than nylon 6,12 and is more rigid due to the fewer number of carbons in its backbone, which leads to its higher $T_{g}$. We observed that cycloaliphatic polyamide is more rigid than its aromatic analogue, while calculations of the free volume through several 
methods indicate that the extent of free volume in cycloaliphatic polyamide is on average, higher. This indicates that the higher $T_{g}$ observed in cycloaliphatic polyamide is predominantly due to rigidity in the backbone, which restricts local reorganization in the available free volume.

\section{Acknowledgements}

This work was supported in part by an appointment to the Postgraduate Research Participation Program at the U.S. Army Research Laboratory (ARL) administered by the Oak Ridge Institute for Science and Education through an interagency agreement between the U.S. Department of Energy and ARL. The authors would like to thank Dr. F.T. Willmore for useful discussion and for use of his CESA program and scripts. Calculations were performed using the DOD Supercomputing Resource Center located at the Air Force Laboratory.

\section{Appendix A}

\section{Calculation of free volume by Bondi Method}

One measure of the chain packing efficiency and the amount of free space in the polymer is the fractional free volume (FFV). A method that is typically used to calculate the FFV at ambient conditions is called the Bondi method[27, 63]. Bondi[63] showed that occupied volume in a polymer can be approximated by 1.3 times the van der Waals volume calculated by group contributions, where the 1.3 factor in this calculation was based on packing density of molecular crystals at $0 \mathrm{~K}$. The FFV was then estimated by taking the ratio of the free volume (specific volume minus the occupied volume) to the specific volume.

The fractional free volume was calculated using the Bondi method, using both the experimental and simulated values of the density (see Table 5). Although the FFV of the nylons are consistently lower than that obtained for our model cycloaliphatic polyamide using both values of density, they are not consistent for nylon 6,6 and 6,12. Deviations between the FFV calculated using these two densities can be due to the formation of crystalline regions, which are indirectly captured in the experimental density but not the simulated density. Crystalline regions 
are ordered and well-packed, so higher levels of crystallinity are associated with lower free volume. We also observe that the cycloaliphatic polyamide has higher fractional free volume than the aromatic polyamides when we utilize the density calculated through simulation. This can in part be due to the difference in size between the cycloaliphatic and aromatic rings present in the backbones of our model polyamides. As mentioned in Section IV D., the van der Waals volume calculated for an cycloaliphatic ring $\left(53.3 \mathrm{~cm}^{3} / \mathrm{mol}\right)$ in our model cycloaliphatic polyamide is larger than that for the aromatic analog $\left(43.3 \mathrm{~cm}^{3} / \mathrm{mol}\right)[27]$.

\begin{tabular}{|c|c|c|}
\hline \multicolumn{3}{|c|}{ Table A. Fractional free volume calculated from Bondi's method } \\
\hline Polymer & \begin{tabular}{|l|} 
Fractional Free \\
Volume using \\
Bondi's Method \\
(Density from \\
Simulation)
\end{tabular} & \begin{tabular}{|l|} 
Fractional Free \\
Volume using \\
Bondi's Method \\
(Density from \\
Experiment)
\end{tabular} \\
\hline Nylon 6,6 & 0.162 & $0.112-0.129$ \\
\hline Nylon 6,12 & 0.191 & 0.098 \\
\hline $\begin{array}{l}\text { Cycloaliphatic } \\
\text { polyamide }\end{array}$ & 0.209 & 0.152 \\
\hline Aromatic Polyamide & 0.181 & $\mathrm{n} / \mathrm{a}$ \\
\hline
\end{tabular}

\section{$\underline{\text { References }}$}

1. Nielsen LE and Landel RF. Properties of Polymers and Composites - Second Edition. New York: Marcel Dekker, Inc., 1994.

2. Daniels CA. Polymers: Structure and Properties. Lancaster: Technomic Publishing Company, Inc., 1989.

3. Assignment of the Glass Transition. Baltimore, MD: ASTM, 1994.

4. Sperling LH. Introduction to physical polymer science, 4th Edition. Hoboken: John Wiley and Sons, 2006.

5. $\quad$ Li M, Liu XY, Qin JQ, and Gu Y. eXPRESS Polymer Letters 2009;3(10):665.

6. Mather BD and Long TE. Hydrogen Bond Functionalized Block Copolymers and Telechelic Oliogmers. Hoboken: John Wiley \& Sons, Inc., 2008.

7. Yamauchi K, Lizotte JR, Hercules DM, Vergne MJ, and Long TE. J. Am. Chem. Soc. 2002;124:8599. 
8. $\quad$ Song PS, Hwang S, and Sheu BC. Cement and Concrete Research 2005;35(8):1546.

9. Vitchuli N, Shi Q, Nowak J, McCord M, Bourham M, and Zhang X. Journal of Applied Polymer Science 2010;116(4):2181.

10. John J, Gangadhar SA, and Shah I. The Journal of Prostetic Dentistry 2002;86(4):424.

11. Tham CY, Tan VBC, and Lee HP. International Journal of Impact Engineering 2008;35(5):304.

12. Tanner D, Fritzgerald JA, and Phillips BR. Advanced Materials 2004;1(5):151.

13. Ahmed D, Hongpeng Z, Haijuan K, Jing L, Yua M, and Muhuo Y. Materials Research 2014;17(5):1180.

14. Cheng SZD, Cao MY, and Wunderlich B. Makromol. Chem. 1988;189:2443.

15. Rastogi R, Vellinga WP, Rastogi S, Schick C, and Meijer HEH. J. Polym. Sci. B Polym. Phys. 2004;42:2092.

16. Yang M, Cao K, Sui L, Qi Y, Zhu J, Waas A, Arruda EM, Kieffer J, Thouless MD, and Kotov NA. ACS Nano 2011;5(9):6945.

17. Leung LM and Koberstein JT. J. Polym. Sci. Polym. Phys. 1985;23:1883.

18. Leung LM and Koberstein JT. Macromolecules 1986;19:706.

19. unpublished data; Glass transition values were measured through a second heating scan at a heating rate of $10 \mathrm{C} / \mathrm{min}$ via differential scanning calorimetry.

20. Gates TS, Odegard GM, Frankland SJV, and Clancy TC. Composites science and technology 2005;65:2416.

21. Mano JF, Gomez Ribelles JL, Alves NM, and Salmeron Sanchez M. Polymer 2005;46:8258.

22. Materials Studio 6.0; Accelrys Software Inc. 2011.

23. Theodorou DN and Suter UW. Macromolecules 1985;18(7):1467.

24. Sun H, Mumby SJ, Maple JR, and Hagler AT. J. Am. Chem. Soc. 1994;116(7):2978.

25. Sun H. J. Phys. Chem. B 1998;102(38):7338.

26. Hockney RW and Eastwood JW. Computer simulation using particles. New York: McGraw-Hill, 1981.

27. van Krevelen DW and Te Nijenhius KT. Properties of Properties. Oxford: Elsevier B.V., 2009.

28. Plimpton S. J. Comp Phys. 1995;117:1.

29. in't Veld PJ, Stone MT, Truskett TM, and Sanchez IC. J. Phys. Chem. B 2000;104(50):12028.

30. Wang X-Y, Willmore FT, Raharjo RD, Wang X, Freeman BD, Hill AJ, and Sanchez IC. J. Phys. Chem. B 2006;110:16685.

31. Jiang Y, Willmore FT, Sanders D, Smith ZP, Ribeiro CP, Doherty CM, Hill AJ, Freeman BD, and Sanchez IC. Polymer 2001;52(10):2244.

32. Willmore FT, Wang X, and Sanchez IC. Journal of Polymer Science: Part B: Polymer Physics 2006;44:1385.

33. Yeh I-C and Wallqvist A. J. Phys. Chem. B. 2009;113:12382.

34. Roux B. Comput. Phys. Commun. 1995;91:275.

35. Yeh I-C, Rinderspacher BC, Andzelm JW, Cureton LT, and La Scala J. Polymer 2014;55:166.

36. Starkweather Jr. HW and Moynihan RE. J. Polym. Sci. 1956;22(102):363.

37. Starkweather Jr. HW, Moore GE, Hansen JE, Roder TM, and Brooks RE. J. Polym. Sci. 1956;21(98):189. 
38. Polymer: A property database, http://www.polymersdatabase.com, last accessed 01/27/2015.

39. Evonik Industries, TROGAMID CX Transparent polyamides iwth an outstanding combination of properties [Materials Data Sheet]. Retrieved from http://www.trogamid.com/sites/dc/Downloadcenter/Evonik/Product/TROGAMID/en/bro chures/Trogamid-CX-en.pdf on 3/30/2015.

40. Qiu W, Habenschuss A, and Wunderlich B. Polymer 2007;48(6):1641.

41. Rios De Anda A, Fillot LA, Rossi S, Long D, and Sotta P. Polym. Eng. Sci. 2011;51:2129.

42. Andrews RJ and Grulke EA. Glass transition temperature of polymers. Hoboken: John Wiley \& Sons, 1999.

43. Komoto H. Rev. Phys. Chem. Jpn. 1967;37:105.

44. Saotome K and Komoto H. J. Polym. Sci., Part A: Polym. Chem. 1966;4:1463.

45. Buchholz J, Paul W, Varnik F, and Binder K. J. Chem. Phys. 2002;117:7364.

46. Kunal K, Robertson CG, Pawlus S, Hahn SF, and Sokolov AP. Macromolecules 2008;41:7232.

47. Javni I, Zhang W, and Petrovic ZS. Journal of Applied Polymer Science 2003;88:2912.

48. Ulrich H. Chemistry and Technology of Isocyanates. New York: John Wiley and Sons, 1996.

49. . In: Salamone JC, editor. Polymeric materials encyclopedia, vol. 3. Boca Raton: CRC Press, 1996.

50. van der Sman RGM. J. Phys. Chem. B 2013;117:16303.

51. Wolkers WF, Oliver AE, Tablin F, and Crowe JH. Carbohydrate Research 2004;339(6):1077.

52. Imamura K, Sakaura K, Ohyama K-I, Fukushima A, Imanaka H, Sakiyama T, and Nakanishi K. J. Phys. Chem. B 2006;110(31):15094.

53. Zhou Q-H, Li M, Yang P, and Gu Y. Macromol. Theory Simul. 2013;22:107.

54. Seymour RW and Cooper SL. Macromolecules 1973;6:48.

55. Paik Sung CS and Schneider NS. Macromolecules 1977;10:452.

56. Wolkenstein MW and Ptitsyn OB. Hydrogen Bonding. New York: Pergamon Press, 1959.

57. $\quad$ Fox Jr TG and Flory PJ. J. Appl. Phys. 1950;21:581.

58. Srivastava A, Alleman C, Ghosh S, and Lee LJ. Modelling Simul. Mater. Sci. Eng. 2010;18:065003.

59. Jean YC, Chen H, Lee LJ, and Yang J. Acta Physica Polonica A 2008;113:1385.

60. Abdel-Hady EE, Mohamed HFM, and El-Sharkawy MRM. Phys. Status Solidi C 2009;6:2420.

61. Kilburn D, Dlubek G, Pionteck J, and Alam MA. Polymer 2006;47:7774.

62. Račko D. Materials Science Forum 2013;733:183.

63. Bondi A. J. Phys. Chem. 1964;68(3):441. 